\title{
Amiodarone for atrial fibrillation: Friend or foe?
}

\author{
Benedict M. Glover, Adrian Baranchuk
}

Heart Rhythm Service, Queen's University and Kingston General Hospital, Kingston, Ontario, Canada

\section{Article p. 622}

Although amiodarone was initially introduced as an antianginal drug, its anti-arrhythmic properties were soon appreciated and over the last four decades it has been used clinically in the management of both atrial fibrillation (AF) and ventricular arrhythmias. Amiodarone has been shown to be superior to both sotalol and propafenone in the maintenance of sinus rhythm in patients with at least one episode of AF [1]. Although some studies have shown some potential benefit of amiodarone in the management of patients with potentially life threatening ventricular arrhythmias, this has not been shown to be a consistent finding $[2,3]$.

The predominant mode of action is class III by inhibiting the IKr and IKs channels resulting in a prolongation of myocardial repolarization homogeneously. It also exhibits class I effects by blocking the sodium channels reducing the conduction velocity, class II effects by blocking the beta-adrenergic receptors and class IV effects by inhibition of the L type calcium channel.

Despite the fact that amiodarone is not and never has been approved by the Food and Drug Administration for the management of AF, it is the most commonly used antiarrhythmic drug used for this purpose [4]. This may be based on the fact that the drug appears to be effective with minimal cardiac contraindications. Unlike several of its competitors, it can be used in patients with impaired left ventricular function as well as coronary artery disease. Although prolongation of the QT interval is commonly seen this results in torsades de pointes in less than $0.5 \%$ of cases $[5,6]$. In fact, the most common cardiac side effect is sinus bradycardia [7].
The major Achilles heel of amiodarone therapy appears to be related to its non-cardiac side effects as a result of tissue penetration and organ toxicity related to the iodine moiety. The list of potential side effects is exhaustive and includes thyroid dysfunction, lung toxicity, inflammatory hepatotoxicity, skin reactions, retinal deposits and pancreatitis. It is therefore good clinical practice to carefully evaluate the potential side effects and discuss these in detail with the patient as well as draw up a monitoring strategy prior to commencing amiodarone.

The manuscript by Qin et al. [8] was a retrospective analysis of patients with structurally normal hearts prescribed anti arrhythmic drugs for the management of AF. Approximately one fifth of these patients were prescribed amiodarone. The remainder were prescribed flecainide, propafenone, sotalol, dofetilide or dronedarone.

Like many studies of this nature, there may be some selection bias and it is noted that patients prescribed amiodarone tended to be more elderly with a higher Charlson comorbidity index. Although propensity matching may help to balance significant differences, caution must still be exercised as several additional differences may still exist which are not adjusted for and which may have been part of the decision process for the physicians when prescribing the anti arrhythmic in the first place. The most important factor of all which was taken into account was to exclude patients with structural heart disease including congestive cardiac failure, coronary artery disease, severe left ventricular hypertrophy and severe valvular heart disease. This helped to maintain a relatively even playing field in terms of the ability to use any of the above mentioned antiarrhythmic drugs. What the

Address for correspondence: Dr Benedict M. Glover and Dr Adrian Baranchuk, Heart Rhythm Service, Queen's University, Kingston General Hospital K7L 2V7, Kingston, Ontario, Canada tel: 6135496666 ext. 3801, fax: 613 548 1387, e-mail: gloverb@kgh.kari.net; barancha@kgh.kari.net

Received: 07.10.2015 Accepted: 17.10 .2015 
authors found was certainly interesting. Firstly and importantly, primary care physicians were more likely to prescribe amiodarone that cardiologists or electrophysiologists. This may reflect a lack of clinical experience with alternative antiarrhythmic agents in this group of physicians. Given the relative cardiac safety of amiodarone, this may be considered an easier therapeutic decision. Cardiologists and electrophysiologists may be more selective in terms of the types of patients who are commenced on amiodarone. This may require further evaluation and education. The fact that there was no significant difference in AF recurrence may reflect the way this information was collected and there is probably insufficient data to draw any further conclusions.

As it would be expected, there was no difference in cardiac deaths between the two groups of patients. There was, however, a higher rate of non-cardiac deaths in the group receiving amiodarone versus those receiving other anti arrhythmic agents. The non-cardiac causes included respiratory failure not related to interstitial lung disease, unspecified neoplasm, pulmonary embolism, dementia, trauma, stroke and a perforated bowel. To the best of our knowledge, none of these are currently considered to be directly related to amiodarone and this may be a general reflection of the patients selected for therapy with amiodarone. Despite this, we strongly recommend that amiodarone should always be used with the utmost caution and that patients are closely monitored for any potential side effects. All current antiarrhythmic drugs have limited efficacy and multiple potential side effects, and we should continue to strive to develop more selective agents with minimal side effects.

Conflict of interest: None declared

\section{References}

1. Waldo AL, Camm AJ, deRuyter H et al. Effect of d-sotalol on mortality in patients with left ventricular dysfunction after recent and remote myocardial infarction. The SWORD Investigators. Survival With Oral d-Sotalol. Lancet, 1996; 348: 7-12.

2. Amiodarone Trials Meta Analysis Investigators. Effect of prophylactic amiodarone on mortality after acute myocardial infarction and in congestive heart failure: Meta-analysis of individual data from 6500 patients in randomised trials. Lancet, 1997; 350: 1417-1424.

3. Boutitie F, Boissel JP, Connolly SJ et al. Amiodarone interaction with beta-blockers: analysis of the merged EMIAT (European Myocardial Infarct Amiodarone Trial) and CAMIAT (Canadian Amiodarone Myocardial Infarction Trial) databases. The EMIAT and CAMIAT Investigators. Circulation, 1999; 99: 2268-2275.

4. Zimetbaum P. Antiarrhythmic drug therapy for atrial fibrillation. Circulation, 2012; 125: 381-389.

5. Kaufman ES, Zimmermann PA, Wang T et al. Risk of proarrhythmic events in the Atrial Fibrillation Follow-Up Investigation of Rhythm Management (AFFIRM) study: A multivariate analysis. J Am Coll Cardiol, 2004; 44: 1276-1282.

6. Riera AR, Uchida AH, Ferreira $\mathrm{C}$ et al. Relationship among amiodarone, new class III antiarrhythmics, miscellaneous agents and acquired long QT syndrome. Cardiol J, 2008; 15: 209-219.

7. Essebag V, Reynolds MR, Hadjis T et al. Sex differences in the relationship between amiodarone use and the need for permanent pacing in patients with atrial fibrillation. Arch Intern Med, 2007; 167: 1648-1165.

8. Qin D, Leef G, Bilal Alam M et al. Mortality risk of long-term amiodarone therapy for atrial fibrillation patients without structural heart disease. Cardiol J, 2015; 22: 622-629. 